\title{
Cryopreservation, actin localization and thermotropic phase transitions in ram spermatozoa
}

\author{
W. V. Holt and R. D. North \\ Institute of Zoology, Zoological Society of London, Regent's Park, London NWI 4RY, UK
}

\begin{abstract}
Summary. The effects of controlled stress, i.e. cooling, upon the distribution of actin in ram spermatozoa were examined to investigate the hypothesis that cytoskeletal proteins are involved in the maintenance of sperm plasma membrane integrity. The normal distribution of actin on the spermatozoon was initially determined. A monoclonal antibody (IgM) interacted exclusively with the post-acrosomal region and the principal piece of the flagellum. By the use of a polyclonal antibody, actin was detected on the acrosome (excluding the equatorial segment), the post-acrosomal region and the whole of the flagellum. The actin was present in its non-filamentous form.

Spermatozoa fixed at $39^{\circ} \mathrm{C}$ and then treated for the immunofluorescent detection of actin with the monoclonal antibody were mostly unstained (proportion stained = $4.4 \%$ ( $\pm 1 \cdot 6$; s.e.m.); $n=8$ ejaculates). Provided spermatozoa were permeabilized by $>0.025 \%$ Triton X-100 before immunofluorescence, actin was localized in the postacrosomal region of all sperm heads, and to a minor extent on the principal piece of the flagellum. Use of the polyclonal antibody confirmed that the post-acrosomal antigen was unmasked by detergent treatment. Slow cooling, over 2-h periods to various temperatures between 5 and $15^{\circ} \mathrm{C}$, also induced an increase in the proportion of cells showing post-acrosomal actin immunoreactivity. Cooling through the temperature range 15 to $10^{\circ} \mathrm{C}$ markedly increased the proportion of immunoreactive cells (mean \pm s.e.m.; $12 \pm 4.5 \%$ at $15^{\circ} \mathrm{C} ; 27 \pm 4.5 \%$ at $10^{\circ} \mathrm{C} ; n=4$ ejaculates). Further cooling to $5^{\circ} \mathrm{C}$ failed to elicit increased staining. Ultrastructural examination of cooled spermatozoa confirmed that a subpopulation of spermatozoa exhibited post-acrosomal actin immunoreactivity after cooling. These results are compatible with the suggestion that actin fulfils a stabilizing function in spermatozoa.
\end{abstract}

Keywords: actin; spermatozoa; membranes; cryopreservation; cytoskeleton; ram

\section{Introduction}

The extent of cryoinjury sustained by mammalian spermatozoa during preservation procedures varies considerably between species, but structural and functional correlates of this diversity have not been identified. White \& Darin-Bennett (1976) and Darin-Bennett \& White (1977) noted that spermatozoa with approximately equal proportions of cholesterol and phospholipid, e.g. human and monkey, were less susceptible to cold-shock than those containing proportionately lower amounts of cholesterol, i.e. bull, ram and boar. They also compared unsaturated:saturated fatty acid ratios between these cold shock-resistant and susceptible species; as the results fell into two groups with ratios of $1: 1$ and 3:1 respectively, it was suggested that high saturated fatty acid content conferred membrane stabilization.

Although cold-shock resistance may be attributable to phenomena involving membrane lipid composition, differences in susceptibility to freezing itself cannot be explained on this basis. For example, whilst boar, bull and ram spermatozoa were grouped together in the analyses cited above, 
the fertility levels after cryopreservation of bull spermatozoa are markedly higher than those of either boar or ram.

In addition to differences in sperm transport mechanisms between species, one possible solution to this discrepancy, suggested by Watson \& Plummer (1985), is that variability in the distribution of membrane-associated cytoskeletal proteins may influence the chilling responses of spermatozoa. Cytoskeletal proteins like actin, tubulin and vimentin have been localized in spermatozoa by a number of research workers (Clarke \& Yanagimachi, 1978; Campanella et al., 1979; Clarke et al., 1982; Virtanen et al., 1984; Ochs et al., 1986; Flaherty et al., 1986) and may stabilize the sperm-head membranes.

Although the articles cited above clearly demonstrated the presence and distribution of cytoskeletal proteins in spermatozoa, functional roles for these proteins are unclear. Involvement of actin in membrane stabilization or remodelling before fertilization has been suggested on several occasions (Welch \& O'Rand, 1985; Peterson \& Hunt, 1987; Saling, 1989). Association of actin with membrane lipids, either directly (St-Onge \& Gicquad, 1990) or via integral membrane proteins (Carraway \& Carraway, 1989) is well recognized. In spermatozoa, actin might therefore contribute to the adhesion between membranes and the underlying cytoplasm; if this were the case, resistance to mechanical and thermal stresses would depend upon the strength of such interactions.

In this study the effects of cooling upon actin distribution in ram spermatozoa were examined as this represents a readily controllable method of imposing mechanical and thermal stress. As normal actin distribution in ram spermatozoa had not previously been described, this was addressed in the initial stages of this work.

\section{Materials and Methods}

Source of semen and experimental technique. Semen was obtained, by the use of an artificial vagina, from Finnish Landrace rams housed at the Royal Veterinary College, London. Before use ejaculates were washed gently to remove seminal plasma. They were diluted 10-fold with temperature-equilibrated Krebs-Henseleit-Ringer's buffered salt solution (KHR; Holt et al., 1988), centrifuged at $500 \mathrm{~g}$ for $10 \mathrm{~min}$, and the sperm pellet resuspended in KHR, at a concentration of approximately $100 \times 10^{6} \mathrm{spermatozoa} / \mathrm{ml}$.

For experiments in which spermatozoa were simply cooled to various temperatures, 2-ml aliquants of diluted spermatozoa were placed in 10-ml capped plastic tubes; these were cooled over 2 -h periods in containers of water in a refrigerator, except when stated otherwise. When spermatozoa were exposed to Triton X-100, the diluted cells were centrifuged $(500 \mathrm{~g} ; 10 \mathrm{~min})$ and resuspended in $1 \mathrm{ml} \mathrm{KHR}$ containing the required detergent concentration. Upon completion of each experiment, the sperm suspensions were fixed for immunofluorescence by the addition of 5 volumes of temperature-equilibrated fixative.

Antibodies. A monoclonal antibody (IgM; code N350) was purchased from Amersham International (Amersham, Bucks, UK). The specificity of the anti-actin antibody was checked by gel electrophoresis and immunoblotting using whole ram spermatozoa as reference material. A protein band of $M_{\mathrm{r}} 43000$ (molecular weight of actin $=43000$ ) specifically cross-reacted with the antibody and was assumed to represent actin. This monoclonal antibody has been extensively characterized in several publications relating to mammalian spermatozoa (for example, Camatini et al., 1986a, b; Flaherty et al., 1986, 1988).

A polyclonal anti-actin antibody (rabbit anti-actin, product No. A-2668; Sigma Chemical Company, Poole, Dorset, UK) was also used to check for the occurrence of actin isoforms not detected by the monoclonal antibody.

Immunofuorescence. Drying of spermatozoa on glass slides was an unsuitable preparative technique for this study as it would, per se, cause membrane damage. An alternative method was therefore adopted, whereby fixed cells in suspension were exposed to antibodies. Spermatozoa were fixed in a mixture containing $3 \%$ paraformaldehyde, $0.1 \%$ glutaraldehyde, $0.1 \mathrm{M}$-phosphate buffer, $\mathrm{pH} 7.3$ and $4 \%$ sucrose (PGPS). Higher glutaraldehyde concentration caused non-specific staining. After fixation for $2 \mathrm{~h}$, the samples were washed in phosphate-buffered saline (PBS; $8.5 \% \mathrm{NaCl}, 50 \mathrm{~mm}$-phosphate buffer, $\mathrm{pH} \mathrm{7.0)}$ ) and small samples of cells (approximately $10 \times 10^{6}$ ) were used for immunofluorescence as outlined below.

The cells were suspended in $50 \mu \mathrm{l}$ of the primary antibody reagent (monoclonal, 1:1000 in PBS; polyclonal 1:40 in PBS) for $30 \mathrm{~min}$ at room temperature, then washed 3 times with PBS and resuspended in the appropriate secondary antibody (FITC-conjugated anti-IgM or FITC-conjugated anti-rabbit IgG; Sigma Chemical Company, Poole, Dorset, UK) for $45 \mathrm{~min}$. Controls were treated with PBS instead of the primary antibody; normal rabbit serum (1:40 in PBS) was used as an additional control for the polyclonal antibody. 
After completion of the immunofluorescence procedure, the cells were washed 3 times in PBS and then resuspended in a glycerol-based compound which inhibits fading of fluorescent dyes (Citifluor; City University, London, UK). A small volume of this suspension $(5-6 \mu$ l) was mounted under a coverslip for fluorescence observation and photomicrography.

Exposure of spermatozoa to Triton X-100. The effect of exposing spermatozoa to graded levels of the detergent Triton X-100 (0.0125\%, 0.025\%, 0.05\%, 0.1\% dissolved in KHR) was tested and compared with controls which had no detergent present. Semen samples from 4 rams (replicates) were washed once in KHR (1:10 dilution and centrifugation), and spermatozoa were added to 2 -ml aliquants of detergent solutions $\left(100 \times 10^{6}\right.$ spermatozoa $\left./ \mathrm{ml}\right)$. After 5 min detergent or control treatment at $39^{\circ} \mathrm{C}$, excess fixative $(4 \mathrm{ml})$ was added to each sample. The cells were washed after fixation for $2 \mathrm{~h}$ and processed for immunofluorescent detection of actin as described above.

Detection of filamentous actin. NBD-phallacidin (7-nitrobenz-2-oxa-1,3-diazolylphallacidin) was obtained from Molecular Probes Inc. (Eugene, Oregon, USA). Smears of washed spermatozoa were fixed in $3.7 \%$ formaldehyde in PBS at room temperature, then washed with PBS. The smears were treated with acetone at $-20^{\circ} \mathrm{C}$ for $5 \mathrm{~min}$, air dried, then stained with NBD-phallicidin reconstituted in PBS after evaporation from the methanolic stock solution.

Electron microscopy and immunocytochemistry. Ram spermatozoa were treated experimentally as described in the text, but for ultrastructural studies not involving immunocytochemistry they were fixed in the PGPS mixture described above, except that $4 \%$ glutaraldehyde concentration was used instead of $0 \cdot 1 \%$. The samples were osmicated and processed for transmission electron microscopy as described previously (Holt \& North, 1984).

For actin immunocytochemistry, spermatozoa were fixed in PGPS ( $0.1 \%$ glutaraldehyde), washed twice in PBS by centrifugation and twice in Tris-buffered saline (TBS; $20 \mathrm{~mm}$-Tris-hydroxymethyl-aminomethane, $0.9 \% \mathrm{NaCl}, 0.1 \%$ bovine serum albumin, $\mathrm{pH} 8 \cdot 2$ ). Aliquants of $100 \times 10^{6}$ spermatozoa were resuspended in the monoclonal anti-actin antibody (1:1000 in TBS) and stored overnight at $5^{\circ} \mathrm{C}$. They were then washed extensively in TBS, and stored overnight at $5^{\circ} \mathrm{C}$ in gold-labelled goat anti-mouse IgM $(15 \mathrm{~nm}$ gold particles, Biocell, Cardiff, UK; $1: 1000$ dilution in TBS). After further extensive washing, the cells were osmicated and processed for electron microscopy. Parallel samples, not incubated in primary antibody, were set up as negative controls.

Scoring procedure and analysis of results. Most of the experiments were set up as randomized block designs with 4 replicates. Since the immunofluorescent results were to be assessed subjectively, the experimental treatments were assigned random codes; the sample tubes were processed in numerical order and the slides examined without knowledge of their identities.

Actin immunofluorescence was scored as either present or absent over the post-acrosomal region; no distinction was made for fluorescent intensity. Each sample of spermatozoa was scored by the following method: 100 spermatozoa were examined on wet-mounted slides using a $\times 100$ oil immersion objective, and every field was viewed with both bright-field and fluorescence illumination. The cells were classified according to presence or absence of fluorescence.

Acrosomal damage was assessed in some experiments. Fixed cells were smeared on agar-coated slides, dried and mounted in distilled water; 100 spermatozoa/treatment were examined using a $\times 100$ oil immersion phase contrast objective and classified as damaged or undamaged on the basis of their acrosomal morphology.

Results of quantitative experiments were expressed as percentages, but transformed to angles for analysis of variance. Individual contrasts within the analyses of variance were examined using Helmert matrices of orthogonal polynomial coefficients.

\section{Results}

\section{Normal localization of actin in ram spermatozoa}

Ram spermatozoa fixed at $39^{\circ} \mathrm{C}$ in PGPS were mostly unstained (proportion stained ( \pm s.e.m. $)=4.4 \pm 1.6 \% ; n=8$ ejaculates) when processed for anti-actir: immunofluorescence (Fig. 1a) using the monoclonal antibody. The stained cells exhibited post-acrosomal fluorescence. With the polyclonal antibody, actin was detected over the principal segment of the acrosome, but the equatorial segment was consistently negative. As with the monoclonal antibody the postacrosomal region was stained in a small proportion of cells (proportion stained ( \pm s.e.m.) $=$ $5 \cdot 7 \pm 0 \cdot 6 \% ; n=4$ ejaculates). The whole length of the flagellum was stained by the polyclonal antibody.

The effect of exposing unfixed spermatozoa to graded levels of the detergent Triton X-100 $(0.0125 \%, 0.025 \%, 0.05 \%, 0 \cdot 1 \%$ dissolved in KHR) was tested and compared to controls with no detergent present, as described in 'Materials and Methods'. Previous studies had established that $0.01 \%$ Triton X-100 caused partial demembranation without complete loss of motility (Vishwanath et al., 1986). Control samples and samples treated with $0.0125 \%$ Triton X-100 were unstained as 

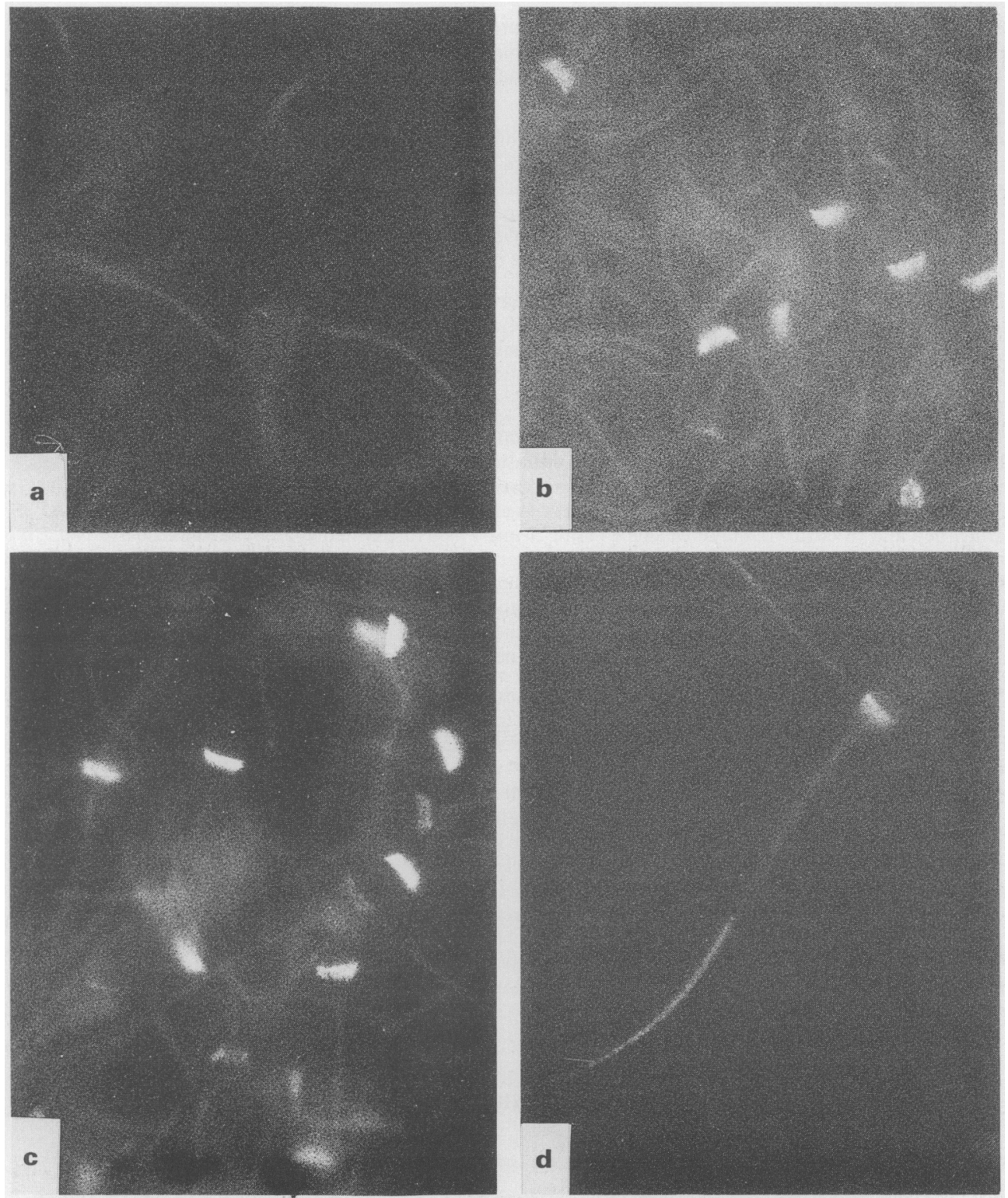

Fig. 1. Wet mounted preparations of ram spermatozoa fixed and processed for actin immunofluorescence. $\times 1000$. (a) Spermatozoa fixed at $39^{\circ} \mathrm{C}$; only background fluorescence is visible. $(b, c)$ Spermatozoa cooled slowly (b) and rapidly (c) to $5^{\circ} \mathrm{C}$ before fixation. Bright postacrosomal fluorescence is visible in some cells; a few cells show weak post-acrosomal fluorescence. (d) A spermatozoon fixed at $39 \mathrm{C}$ after 5 -min incubation in $0.025 \%$ Triton X-100. Actin immunoreactivity is visible over the post-acrosomal region and the principal-piece of the flagellum.

before when monitored with the monoclonal antibody. However, $100 \%$ of spermatozoa pretreated with $0.025 \%, 0.05 \%$ or $0.1 \%$ Triton X-100 showed bright fluorescence over the post-acrosomal region and the principal piece of the flagellum (Fig. 1d). This result, showing that actin is accessible to antibody after a mildly disruptive treatment, was reproduced using the polyclonal antibody. Here the proportions of spermatozoa showing post-acrosomal staining after treatment in control 
$(0 \%), 0.0125 \%, 0.025 \%$ and $0.05 \%$ Triton $X-100$ were $5 \cdot 7,62,94$ and $100 \%( \pm 10 \cdot 3$ s.e.m. $)$ respectively.

Spermatozoa treated with NBD-phallicidin were unstained despite the extensive permeabilization treatment procedure.

\section{Localization of actin in cooled spermatozoa}

Having established that actin was not demonstrable unless a membrane-disruption treatment was applied first, experiments were set up to determine whether low temperature also induced the exposure of actin to external antibodies. The following experiments were evaluated using the monoclonal antibody only, since the presence or absence of post-acrosomal fluorescence could be observed and scored unequivocally.

A block-design experiment with 4 replicates (rams) and 4 treatments was set up to begin exploration of this question. Spermatozoa were cooled slowly $\left(0 \cdot 25^{\circ} \mathrm{C} / \mathrm{min}\right)$ and quickly $(0 \cdot 2-\mathrm{ml}$ aliquants in glass tubes plunged into $5^{\circ} \mathrm{C}$ water) to $5^{\circ} \mathrm{C}$, and compared with samples fixed at $39^{\circ} \mathrm{C}$ immediately (fast-cooling control) or after incubation for $2 \mathrm{~h}$ at $39^{\circ} \mathrm{C}$ (slow-cooling control). Cells fixed at $39^{\circ} \mathrm{C}$ were unstained after processing for actin immunofluorescence, but those cooled to $5^{\circ} \mathrm{C}$ were brightly stained over the post-acrosomal region (Fig. $1 \mathrm{~b} \& \mathrm{lc}$ ). This was a preliminary experiment and quantitative assessment of the results was not undertaken.

Correlative estimates of sperm motility were subjectively performed on the samples used in this experiment; for this purpose the cooled samples were rewarmed to $39^{\circ} \mathrm{C}$ for examination. After fast cooling and rewarming the proportions of motile cells in the 4 replicates were subjectively scored as $1 \%, 1 \%, 20 \%$ and $50 \%$ respectively. The quality of progressive motility was minimal. The equivalent replicate values for the slow-cooled cells were $60 \%, 50 \%, 40 \%$ and $70 \%$, whilst the quality of motility was very similar to that observed before cooling and after $2-\mathrm{h}$ incubation at $39^{\circ} \mathrm{C}$. Mean motility scores for the two groups (18 and $55 \%$ respectively) were not statistically different, although two individual samples were severely damaged by the rapid-cooling treatment.

Both slow and fast cooling induced exposure of actin. However, in contrast to the findings with Triton X-100, no antibody binding occurred on the principal piece of the flagellum after cooling. It was also evident that the actin isoform recognized by the monoclonal antibody did not undergo topographical redistribution after cooling. Moreover, the exposure of antibody-reactive sites in slowly cooled spermatozoa could not be equated with severe plasma membrane damage since a large proportion of the cells maintained their motility, especially if cooling was performed slowly.

Since actin became accessible to antibody after cooling to $5^{\circ} \mathrm{C}$, and apparently without necessarily involving membrane damage severe enough to immobilize the cells, it was of interest to determine whether a temperature threshold exists at which this effect is induced. This question was addressed in further experiments.

The first was a randomized block experiment with 5 treatments (final temperatures) and 4 replicates (rams). Spermatozoa were cooled from $39^{\circ} \mathrm{C}$ to $16.5^{\circ} \mathrm{C}, 12.5^{\circ} \mathrm{C}$ and $11^{\circ} \mathrm{C}$ over a 2 -h period; there were 2 control treatments, i.e. (1) spermatozoa maintained at $39^{\circ} \mathrm{C}$ for $2 \mathrm{~h}$, and (2) spermatozoa fixed at $39^{\circ} \mathrm{C}$ immediately after dilution in $\mathrm{KHR}$. Incubation for $2 \mathrm{~h}$, without temperature reduction, had no effect upon the low incidence of actin-positive spermatozoa (Fig. 2a; compare ' $\mathrm{F}$ ' and 'Inc'). Cooling to $12.5^{\circ} \mathrm{C}$ or $16.5^{\circ} \mathrm{C}$ increased $(P<0.05)$ the incidence of fluorescent-stained spermatozoa from $5 \%$ (control) to approximately $15 \%$. At $11^{\circ} \mathrm{C}$ the incidence of stained spermatozoa was 5 times higher than the control values $(P<0.05)$, i.e. $27 \%$ ( $\pm 5 \cdot 0$ s.e.m.). These results are summarized in Fig. 2(a).

To check that the increase in proportion of fluorescent spermatozoa induced by cooling from 12.5 to $11.0^{\circ} \mathrm{C}$ was reproducible, another experiment was undertaken using a slightly different range of target temperatures. This was a randomized block with 4 replicates (rams) and 6 treatments, i.e. temperatures $\left(21.5^{\circ} \mathrm{C}, 15^{\circ} \mathrm{C}, 12^{\circ} \mathrm{C}, 10^{\circ} \mathrm{C}\right.$ and $5^{\circ} \mathrm{C}$ and control cells; $39^{\circ} \mathrm{C}$ for $2 \mathrm{~h}$ ). The results are summarized in Fig. 2(b). As before, cells fixed after incubation at $39^{\circ} \mathrm{C}$ were mostly 


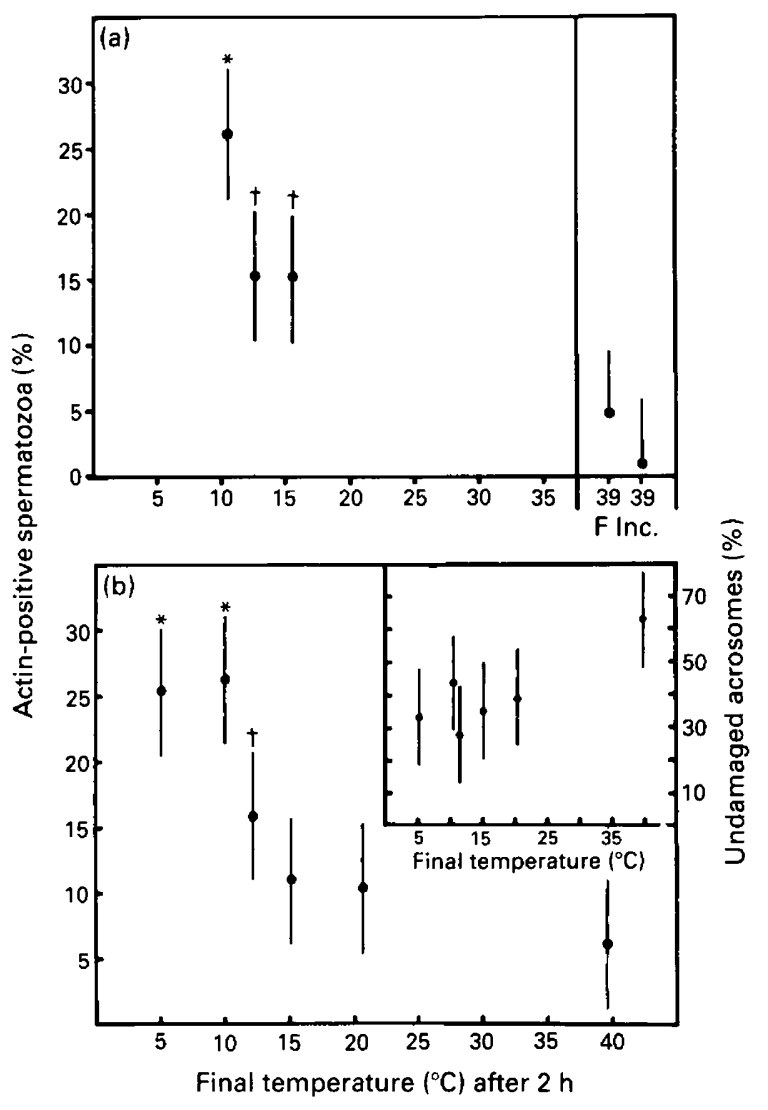

Fig. 2. Two similar experiments in which spermatozoa were cooled over a 2-h period to various temperatures ( $x$ axis); then fixed, processed for actin immunoreactivity and scored for percentage actin-positive spermatozoa ( $y$ axis). Each point is the mean ( \pm s.e.m. from analysis of variance) of 4 replicates. In (a) samples are represented which were fixed at $39^{\circ} \mathrm{C}$ at the beginning of the experiment (F) and incubated at $39^{\circ} \mathrm{C}$ for $2 \mathrm{~h}$ before fixation (Inc). *Value higher than all other points $(P<0.005)$. + Values higher than unlabelled points $(P<0.05)$. In (b), * = values higher than all other points $(P<0.05)$, and $+=$ values higher than unlabelled points $(P<0.025)$. Inset: Mean ( \pm s.e.m. from analysis of variance) of percentage undamaged acrosomes $(y$ axis) $v s$ final temperature after cooling. No statistically significant differences between any of the values.

unstained ( $7.0 \pm 4.5 \%$ (s.e.m.) positive cells). Cooling to $15^{\circ} \mathrm{C}$ did not elicit a significant increase in the proportion of stained cells. At $12^{\circ} \mathrm{C}$ a higher proportion (16.8 $\pm 4.5 \%$ (s.e.m.) positive cells) was indeed apparent $(P<0.025)$. The proportion of stained cells was further elevated by cooling to $10^{\circ} \mathrm{C}(P<0.025)$, at which a maximal response $(27.5 \pm 4.5 \%$ (s.e.m.) positive cells) was obtained as before. Further cooling to $5^{\circ} \mathrm{C}$ produced no more increase in the proportion of stained cells. Correlative assessment of acrosomal damage in this experiment was undertaken. Cooling effects were not statistically confirmed, but from gross examination of the data (Fig. 2b; inset) it appeared that cooling to any of the target temperatures caused approximately $20 \%$ acrosomal damage.

\section{Ultrastructural localization of actin}

To determine more precisely the sites of actin distribution in ram spermatozoa, cells from 2 rams were either incubated at $39^{\circ} \mathrm{C}$ for $2 \mathrm{~h}$, or cooled to $5^{\circ} \mathrm{C}$ over the same period, then fixed and processed as described above. 


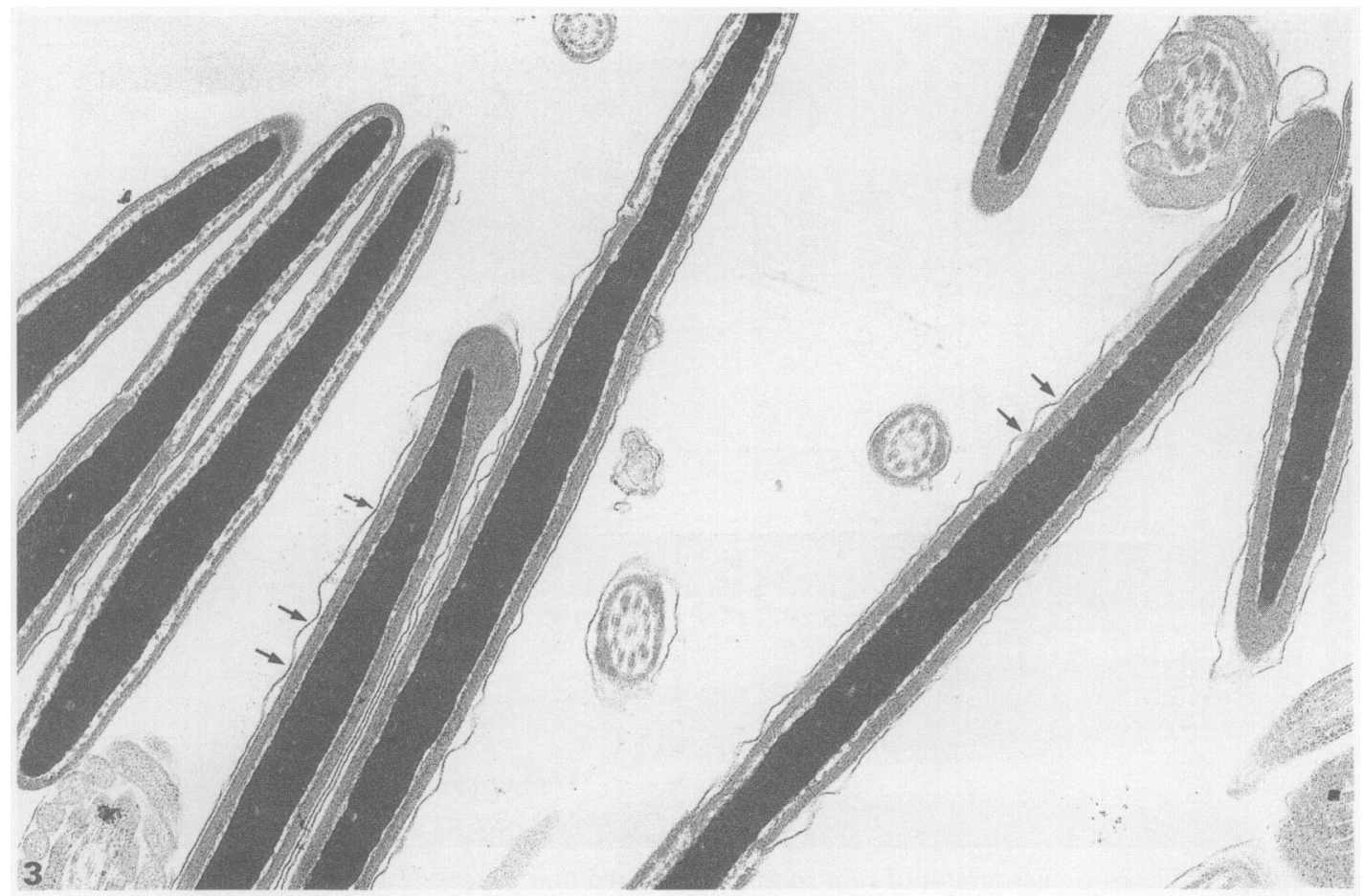

Fig. 3. Electron micrograph of ram spermatozoa cooled to $5^{\circ} \mathrm{C}$ over $2 \mathrm{~h}$ before fixation in PGPS (4\% glutaraldehyde). Spermatozoa do not exhibit significant damage, although plasma membranes have become separated from the outer acrosomal membrane. Arrows indicate points of possible attachment between plasma membranes and the outer acrosomal membrane. $\times 22500$.

Plasma membrane integrity was well preserved in spermatozoa not subjected to cooling. Incubation at $39^{\circ} \mathrm{C}$ for $2 \mathrm{~h}$ failed to induce morphological changes in the acrosomes, which were almost exclusively intact. Post-acrosomal domains of the sperm heads were also undamaged and bounded by the plasma membrane. The majority of cooled spermatozoa were not overtly damaged; in most cases, however, the plasma membrane overlying the acrosome was distanced from the outer acrosomal membrane, as if swelling had occurred, except in discrete sites of possible attachment (Fig. 3; arrows). A proportion of spermatozoa had clearly undergone more extensive damage; in these cells the plasma membrane was missing over all or part of the sperm head, and in some the postacrosomal matrix was disrupted. Actin-immunoreactive sites were apparent on the post-acrosomal surfaces of a minority of cells after cooling (Figs 4 \& 5), and none was detected in uncooled spermatozoa. Quantitative assessment of the proportion of immunoreactive cells was not undertaken, but it was judged to be within the range of $20-40 \%$ seen by light microscopy. Poor plasma membrane preservation was obtained after fixation in low-concentration glutaraldehyde; however, damaged and undamaged cells were readily discriminated and it was clear that only cells with damaged or missing post-acrosomal plasma membranes were labelled with gold particles. Where more extensive disruption of the post-acrosomal matrix had occurred, gold particles had penetrated into the resultant spaces between matrix material and the nuclear membrane (Fig. 5).

\section{Discussion}

The experiments described here revealed that actin is present in most regions of the ram spermatozoon. The post-acrosomal region, however, contains a distinct actin isoform not found elsewhere 


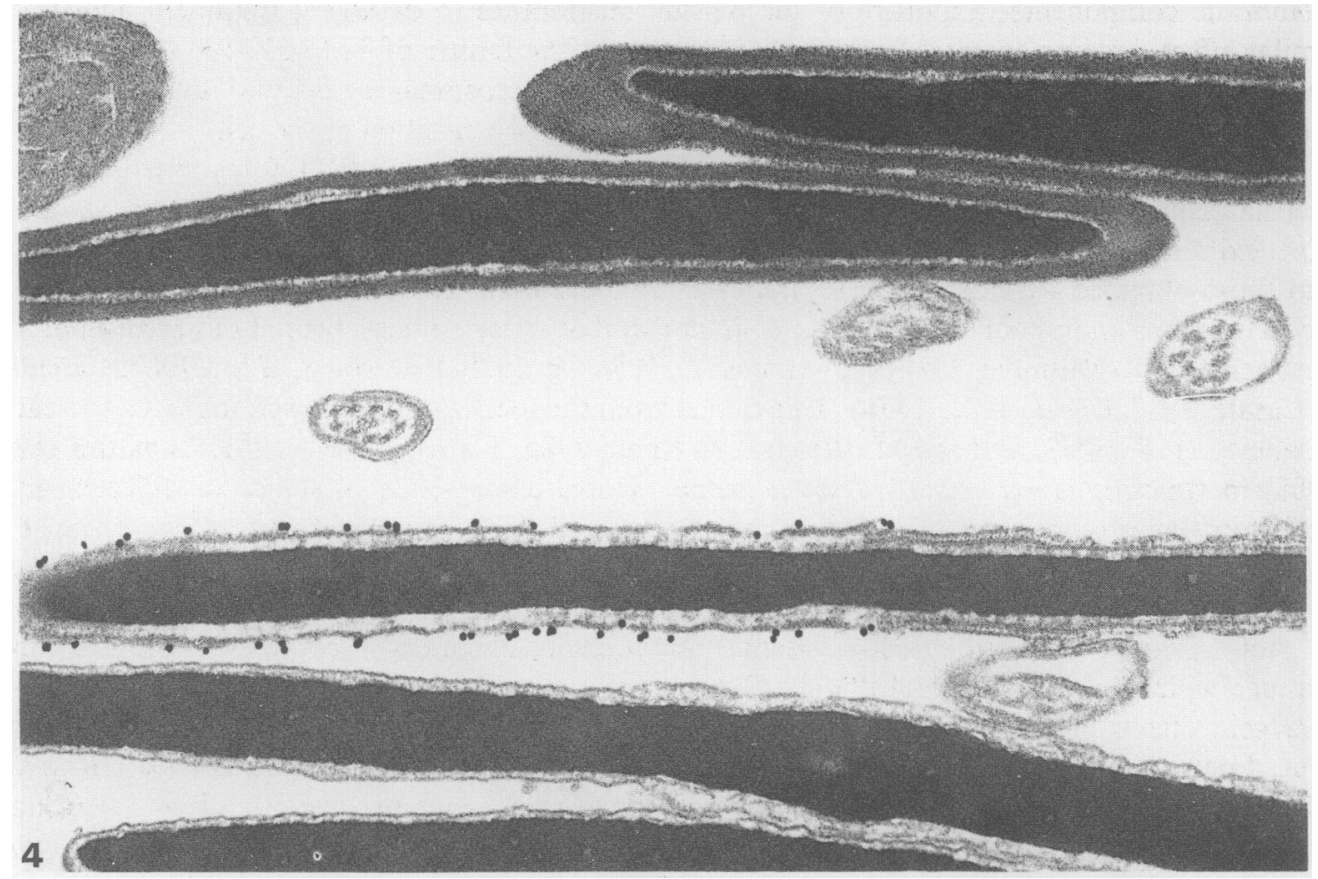

Fig. 4. Electron micrograph of ram spermatozoa fixed and processed for cytochemical detection of actin; electron-dense gold particles represent sites of immunoreactivity. Actin is situated exclusively and evenly over the post-acrosomal region of the sperm head. $\times 42000$.

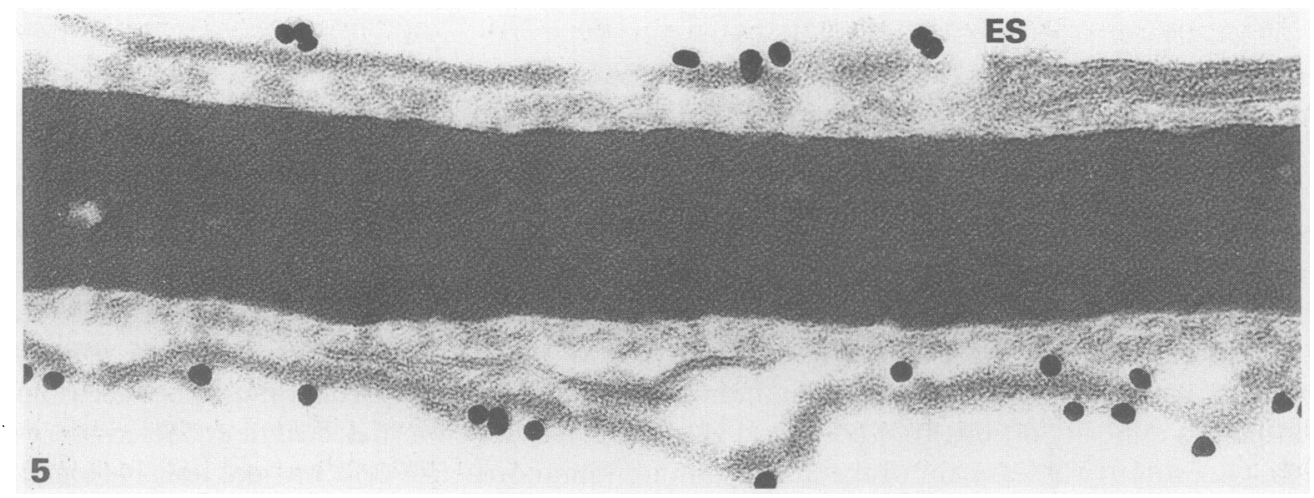

Fig. 5. Electron micrograph of ram spermatozoa fixed and processed for cytochemical detection of actin. Electron-dense gold particles, representing sites of actin-immunoreactivity are situated on the surface of the post-acrosomal region, but near to the equatorial segment (ES) of the acrosome they have penetrated the post-acrosomal matrix. $\times 113000$.

on the sperm head. Moreover, actin in this region is normally masked and only accessible to extracellular antibodies after a mild disruptive treatment. This is consistent with a submembranous or indeed intramembranous distribution.

Cooling the spermatozoa affected the plasma membrane, allowing progressively more spermatozoa to become immunoreactive as the temperature was lowered. This could have occurred through reorientation of the antigen within the plasma membrane or through partial loss of plasma 
membrane components. Exposure of the plasma membranes to detergent treatment achieved a similar effect, lending support to the latter argument. The failure of Triton X-100 to remove the antigen indicates that actin is firmly anchored to the post-acrosomal region, and is consistent with the hypothesis that its function is related to the structural organization of the cell.

Given that cellular integrity and function is maintained inconsistently after cryopreservation of spermatozoa from different species, variability in cytoskeletal protein distribution would be expected if they are important in governing structural stability. Comparison of the present results and those obtained with other species indicates that actin localization may vary between species. Actin distribution in boar spermatozoa is similar to that reported here, being both acrosomal and post-acrosomal (Tamblyn, 1980; Camatini et al., 1986a, b, 1988; Peterson \& Hunt, 1987; Camatini \& Casale, 1987; Casale et al., 1988). This differs from the localization reported for domestic cattle (Flaherty et al., 1988) and rabbit (Welch \& O'Rand, 1985; Flaherty et al., 1986; Camatini et al., 1988 ) in which it is exclusively post-acrosomal. Unfortunately, the existence of different actin isoforms in various regions of the sperm head confounds these comparisons to some extent. For example, the Amersham monoclonal antibody, used in this and other studies, appears to have a specific affinity for the post-acrosomal form of the antigen.

Actin availability on the post-acrosomal region changed during cooling, by responding in a manner which broadly resembled the effects of mild detergent treatment. Previous studies on detergent effects (for example, Vishwanath et al., 1986) established that $0.01 \%$ Triton X-100 caused partial demembranation without complete loss of motility. Simple tests carried out in this laboratory demonstrated that $0.01 \%$ Triton X-100 caused ram spermatozoa to adopt a semi-rigid 'bow-like' configuration, whilst retaining the ability to generate flagellar beats. This effect could also be induced in some cells by cooling to $12^{\circ} \mathrm{C}$, and was reversed by the addition of $2 \mathrm{~mm}$ adenosine triphosphate (ATP) to the medium (W. V. Holt, unpublished). The main effects of cooling were probably mediated through changes in plasma membrane permeability, with different protein populations becoming sequentially accessible to externally situated antibodies. This would be compatible with exposure of actin sites caused by changes in the physical state of membrane lipids and domains.

Membrane permeability, membrane-bound enzyme activity and normal cellular functions are dependent upon regulation of membrane lipid composition and fluidity (for reviews, see Quinn, 1981; Tocanne et al., 1989). Thermotropic lipid phase transitions are therefore likely contributors to cryoinjury during cooling as membrane lipids undergo physical transitions from fluid to gel phases at characteristic temperatures. If the physical state of the plasma membrane changes during cooling, cellular integrity may be compromised. Holt \& North (1986) showed that such phase transitions do indeed occur in purified ram sperm plasma membranes; one was detected at $17^{\circ} \mathrm{C}$ and another at $22^{\circ} \mathrm{C}$. More recently, by the use of Fourier transform infrared spectroscopy, mid-point transition temperatures of 13.6 and $21.1^{\circ} \mathrm{C}$ have been detected in human and goat spermatozoa respectively (Crowe et al., 1989). As these phase transitions involve complex lipid interactions, the mid-point values cannot be regarded as switching points between stable and unstable states. However, they are close to the critical range reported here for the induction of membrane instability, and support the view that perturbation of membrane fluidity is partly responsible for cryoinjury at temperatures above freezing.

The heterogeneity of the sperm surface (for review, see Holt, 1984) and indeed of the sperm population, implies strongly that biophysically derived phase transition points provide an intricately derived average value. The present results are therefore useful in revealing specific information about phase transitions of the post-acrosomal region. Coincidentally, the critical range for membrane permeabilization found in this study is identical to one found by cryomicroscopy (Holt et al., 1988), whereby live ram spermatozoa exhibited specific flagellar damage when cooled through the temperature range $10-14^{\circ} \mathrm{C}$.

The scoring system used for quantifying post-acrosomal actin in spermatozoa produced figures for the proportion of cells displaying immunoreactivity to the actin antibody, with individual cells 
changing their status at slightly different temperatures within the critical range. Maximal postacrosomal staining was evident after cooling to $10^{\circ} \mathrm{C}$, but approximately $70 \%$ of spermatozoa remained unstained upon further cooling. These cells are clearly not unstained because they do not contain actin, but may represent the live, undamaged, population which retained motility and acrosomal integrity. If this is indeed the case, the members of the sperm population undergoing membrane damage during chilling must differ from other cells in the ejaculate with regard to membrane structure, composition, and ability to undergo lipid-phase transitions. Functionally distinct subpopulations of spermatozoa result from mixing during maturation in the epididymis, and undergo capacitation asynchronously over a prolonged period both in vitro and in vivo, and so it is no surprise to find a subpopulation especially vulnerable to cryoinjury. It will be of interest to determine the nature of the subpopulation so identified.

Although further work will be required to establish the relationship between post-acrosomal actin and overlying plasma membrane proteins, the possibility exists that actin fulfils a stabilization role. Independent evidence supports the view that actin, at least in the post-acrosomal region of rabbit spermatozoa, stabilizes plasma membrane proteins and may even have a role in zona pellucida-sperm binding (Welch \& O'Rand, 1985; Saling, 1989). The post-acrosomal rabbit sperm antigen in question (rabbit sperm autoantigen; O'Rand et al., 1986) does not show lateral mobility and is thought to be anchored to underlying non-filamentous actin.

Actin-membrane protein interactions may become destabilized during cooling. Freeze-fracture experiments on ram spermatozoa demonstrated clearly that some post-acrosomal membrane proteins (intramembranous particles) undergo lateral redistribution in response to the same cooling conditions as used in the present study (Holt \& North, 1984). Disruption of actin interactions during cooling and freezing could have far-reaching consequences for the subsequent fertility of spermatozoa. Clearly there is a strong likelihood that comparative cytoskeletal protein distribution between species, by no means a consistent pattern, is of significance in governing susceptibility to cryoinjury.

We are grateful to Dr P. F. Watson (Royal Veterinary College, London) for supplying the ram semen used here. The studies were supported by the Medical Research Council and the Agriculture and Food Research Council.

\section{References}

Camatini, M. \& Casale, A. (1987) Actin and calmodulin coexist in the equatorial segment of ejaculated boar spermatozoa. Gamete Res. 17, 97-105.

Camatini, M., Anelli, G. \& Casale, A. (1986a) Identification of actin in boar spermatids and spermatozoa by immunoelectron microscopy. Eur. J. Cell Biol. 42, 311-318.

Camatini, M., Casale, A., Anelli, G. \& Cifarelli, M. (1986b) Immunoelectronmicroscopic localization of actin in ionophore-treated boar sperm. Cell Biol. Int. Reports 10, 231-238.

Camatini, M., Casale, A., Cifarelli, M. \& Valenti, R. (1988) Actin and calmodulin localization during boar spermiogenesis. In Cell Interactions and Differentiation, pp. 223-235. Ed. G. Ghiara. University of Naples, Naples.

Campanella, C., Gabbiani, G., Baccetti, B., Burrini, A. G. \& Pallini, V. (1979) Actin and myosin in the vertebrate acrosomal region. J. submicr. Cytol. 11, 53-71.

Carraway, K.L. \& Carraway, C.A.C. (1989) Membranecytoskeleton interactions in animal cells. Biochim. Biophys. Acta 988, 147-171.
Casale, A., Camatini, M., Skalli, O. \& Gabbiani, G. (1988) Characterization of actin isoforms in ejaculated boar spermatozoa. Gamete Res. 20, 133-144.

Clarke, G.N. \& Yanagimachi, R. (1978) Actin in mammalian sperm heads. J. exp. Zool. 205, 125-132.

Clarke, G.N., Clarke, F.M. \& Wilson, S. (1982) Actin in human spermatozoa. Biol. Reprod. 26, 319-327.

Crowe, J.H., Folkert, A., Hoekstra, L., Crowe, N., Achordoguy, T.J. \& Drobnis, E. (1989) Lipid phase transitions measured in intact cells with Fourier transform infra red spectroscopy. Cryobiology 26, 76-84.

Darin-Bennett, A. \& White, I.G. (1977) Influence of cholesterol content of mammalian spermatozoa on susceptibility to cold-shock. Cryobiology 14, 466-470.

Flaherty, S., Winfrey, V.P. \& Olson, G.E. (1986) Localization of actin in mammalian spermatozoa: a comparison of eight species. Anat. Rec. 216, 504-515.

Flaherty, S., Winfrey, V.P. \& Olson, G.E. (1988) Localization of actin in human, bull, rabbit and hamster sperm by immunoelectron microscopy. Anat. Rec. 221, 599-610. 
Holt, W.V. (1984) Membrane heterogeneity in the mammalian spermatozoon. Int. Rev. Cytol. 87, 159-194.

Holt, W.V. \& North, R.D. (1984) Partially irreversible cold-induced lipid phase transitions in mammalian sperm plasma membrane domains: freeze-fracture study. J. exp. Zool. 230, 473-483.

Holt, W.V. \& North, R.D. (1986) Thermotropic phase transitions in the plasma membrane of ram spermatozoa. J. Reprod. Fert. 78, 445-457.

Holt, W.V., Morris, G.J., Coulson, G. \& North, R.D. (1988) Direct observation of cold-shock effects in ram spermatozoa with the use of a programmable cryomicroscope. J. exp. Zool. 246, 305-314.

Ochs, D., Wolf, D.P. \& Ochs, R.L. (1986) Intermediate filament proteins in human sperm heads. Expl Cell Res. 167, 495-504.

O'Rand, M.G., Welch, J.E. \& Fisher, S.J. (1986) Sperm membrane and zona pellucida interactions during fertilization. In Molecular and Cellular Aspects of Reproduction, pp. 131-143. Eds D. S. Dhindsa \& O. P. Bahl. Plenum Publishing Co., New York.

Peterson, R.N. \& Hunt, W.P. (1987) Evidence for the presence of F-actin in boar sperm plasma membranes. Biol. Reprod. 36 (Suppl. 1), Abstr. 288.

Quinn, P.J. (1981) The fluidity of cell membranes and its regulation. Biophys. molec. Biol. 38, 1-104.

Saling, P.M. (1989) Mammalian sperm interaction with extracellular matrices of the egg. Oxford Rev. Reprod. Biol. 11, 339-388.
St-Onge, D. \& Gicquad, C. (1990) Research on the mechanisms of interactions between actin and membrane lipids. Biochem. Biophys. Res. Commun. 167, 40-47.

Tamblyn, T.M. (1980) Identification of actin in boar epididymal spermatozoa. Biochem. Biophys. Res. Commun. 62, 328-335.

Tocanne, J-F., Dupou-Cezanne, L., Lopez, A. \& Tournier, J-F. (1989) Lipid lateral diffusion and membrane organization. FEBS Lett. 257, 10-16.

Virtanen, I., Badley, R.A., Paasivuo, R. \& Lehto, V.P. (1984) Distinct cytoskeletal domains revealed in sperm cells. J. Cell Biol. 99, 1083-1091.

Vishwanath, R., Swan, M.A. \& White, I.G. (1986) Effect of triton X-100 on ultrastructure, reactivation, and motility characteristics of ram spermatozoa. Gamete Res. 15, 361-371.

Watson, P.F. \& Plummer, J.M. (1985) Responses of boar sperm membranes to cold shock and cooling. In Deep Freezing of Boar Semen, pp. 113-127. Eds L. A. Johnson \& K. Larsson. Swedish University of Agric. Sci., Dept. Obstetrics \& Gynaecology, Uppsala.

Welch, J.E. \& O'Rand, M.G. (1985) Identification and distribution of actin in spermatogenic cells and spermatozoa of the rabbit. Devl Biol. 109, 411-417.

White, I.G. \& Darin-Bennett, A. (1976) The lipids of sperm in relation to cold shock. Proc. 8 th Int. Congr. Anim. Reprod. \& AI Krakow, pp. 951-954.

Received 16 May 1990 